\title{
A Ubiquitous Context-Aware Environment for Surgical Training
}

\author{
P. Ordóñez, P. Kodeswaran, V. Korolev, W. Li, O. \\ Walavalkar, B. Elgamil, A. Joshi, T. Finin, Y. Yesha \\ Department of Computer Science and Electrical Engineering \\ University of Maryland, Baltimore County \\ Baltimore, MD USA \\ \{ ordopa1, palanik1, vkorol1, wenjia1, onkar1, ben8, joshi, \\ finin, yeyesha\}@cs.umbc.edu
}

\begin{abstract}
The age of technology has changed the way that surgeons are being trained. Traditional methodologies for training can include lecturing, shadowing, apprenticing, and developing skills within live clinical situations. Computerized tools which simulate surgical procedures and/or experiences can allow for "virtual" experiences to enhance the traditional training procedures that can dramatically improve upon the older methods. However, such systems do not to adapt to the training context. We describe a ubiquitous computing system that tracks low-level events in the surgical training room (e.g. student locations, lessons completed, learning tasks assigned, and performance metrics) and from these derive the training context. This can be used to create an adaptive training system.
\end{abstract}

Keywords- context awareness; ubiquitous computing; surgical training.

\section{INTRODUCTION}

Context aware ubiquitous computing systems must process streams of data arriving from sensors, services, devices and other systems to construct and maintain a model of their environment. If the environment is complex, the volume of data will be large and if the system aspires to be intelligent, the processing over the data may be computationally expensive. In ongoing research, we are designing and implementing a framework for constructing intelligent, context-aware ubiquitous computing systems.

We are pursuing the general technical goals while working with colleagues at the University of Maryland Medical Center (UMMC) to use an evolving project to implement a system named CAST, Context-Aware Surgical Training. CAST is part of the Operating Room of the Future (ORF) [15] project that is housed in the newly opened Maryland Advanced Simulation, Training, Research, and Innovation Center (MASTRI). It is a facility with authentic operating rooms specially renovated/constructed and instrumented to support innovative research and training. We have already constructed and deployed a partial prototype of the CAST system in the MASTRI Center to test the feasibility of our approach, which is described in this paper.

This work is supported in part by award W81XWH-06-2-0057 from USAMRMC/TATRC. The views/opinions/findings are those of the authors alone and not the supporting agency.

\author{
I. George \\ Department of General Surgery \\ University of Maryland Medical Center \\ Baltimore, MD USA \\ igeorge@smail.umaryland.edu
}

\section{THE CAST VISION: BACKGROUND AND MOTIVATION}

Traditionally, surgical training has consisted of the resident shadowing senior surgeons and practicing diagnostic and procedural skills on live patients. In 1999, Gorman et al. [4] stated that estimated costs of training chief residents for general surgery alone cost $\$ 53$ million per year. A long standing debate over the ethics and practicality of such practices is also of concern [2]. Furthermore, statistics like the following demonstrate the need for a dramatic change in clinical pedagogy. A survey of residents and faculty in surgical training programs in 2003 described that more than $87 \%$ of the 1,653 responses from residents surveyed indicated that they had an 80 hour work week. $45 \%$ reported working more than 100 hours per week. $57 \%$ reported that their cognitive abilities had been impaired by fatigue [5]. Furthermore, although apprenticeships have been shown to be very effective, in the case of a surgical procedure, the well being of a patient outweighs the training of the resident.

Computer-enhanced simulations show promise for addressing all of these concerns. However, as Granger [7] states in his dissertation, "The key issue is not whether to creep forward through evolution of digital substitutes, but whether to promote the revolution of clinical practice through the integration of pervasive computing technologies."

Our system aims to improve the training provided by simulators by making them a part of a context-aware training environment. This allows the training process to require less direct intervention from mentors in many of the routine tasks. We aim to reason over sensed data streams to infer context about the events in the training process. In the initial prototype system described in this paper, we focus on laparoscopic surgical training ${ }^{1}$. We track the presence of surgical residents in the training rooms, which training machine they have used and for how long, which lessons they have downloaded etc. This information is then used to guide the students to the practice/tests they need to take. Similarly, recordings of students' hands as they use the laparoscopic trainers as well as the output from the simulators are made available to instructors, who can then view and analyze them, add comments and annotations, and suggest skills on which the

${ }^{1}$ Laparoscopic surgery involves operations in the abdomen that are performed through small incisions rather than the larger ones required by traditional surgical procedures. 
trainee needs to focus. Instructor feedback and suggestions can be automatically provided to trainees as podcasts or text messages.

An Electronic Student Record (ESR) provides a centralized repository of student information and progress, and helps infer their appropriate pedagogic context. This record is described in the semantic web language RDF-S, but is presently implemented as a database schema. The ESR will provide a comprehensive summary of the students' progress such as the time spent at each machine, chapters checked out, video captures etc. which will help the instructor to review student performance without physically being present in the training room.

In this system, the student benefits from the guiding elements that can be brought to bear and the real time adaptation of the training. Moreover, the trainer is now able to change their curriculum to meet the needs of their students. For the patient, the movement to disrupt the old and oft-repeated mantra of "See one, do one, teach one" is quite telling. In particular, the steep learning curves of new surgical technologies can now be mastered by trainees outside of live operative settings.

\section{RELATED WORK}

"William Osler wrote in 'The Principles and Practice of Medicine' in 1982 that: 'To learn medicine without books is to sail an uncharted sea, while to learn medicine only from books, is not to go to sea at all."' [7]. In the $21^{\text {st }}$ century, the question is can we virtually go to sea?

\section{A. Virtual Reality Training}

Parallels have been drawn between pilots and surgeons in that both must be able to respond to potentially life-threatening situations in unpredictable environments [4]. A pilot must be prepared to land a plane when several engines have failed and a surgeon must be able to respond to a cardiac arrest in the middle of open heart surgery. Flight simulators have long been used to train pilots for the worst of circumstances. In fact, the simulators of today are so effective that they are often used to train a pilot on a new version of a plane, and the pilot flies the real plane on a scheduled flight [10].

As a result, surgical simulation is rapidly becoming the standard for surgical training. Training simulations currently exist for endoscopic sinus surgery [3], ossiculoplasty surgery [8], and orthopedic surgery [7] to name a few. Many of these simulations create a virtual reality using video gaming technology. A recent paper in 2007 correlated video gaming skills with the laparoscopic surgical skills [12], although that should not be a reason to relax concerns about the amount of time children spend playing video games.

Some of the aforementioned systems use multimedia and hypermedia to enhance surgical training [7]. Others simply use 2-D video and haptic devices as in most video games [3][8]. Others use a hybrid approach where they combine the 2-D video with a visual awareness of objects and events in a room [11]. Welch et al. are capturing and displaying high-fidelity 3$\mathrm{D}$ graphical reconstructions of the actual time-varying events for the purpose of doing on-line consultation and off-line surgical training [9]. This research could help to provide surgical training and mentoring by specialists to generalist doctors in isolated hospitals in developing countries [6].

The 3-D graphical reconstructions are being stored in Immersive Electronic Books (IEB) for surgical training. Via IEB surgeons can explore previous surgical treatments in 3-D [10]. Thus, in the same way, a pilot can test out a new version of a plane time and time before she flies it, a surgeon can see a surgical procedure and interact with it time and time again until she performs it.

\section{B. Other Training}

B-line Medical [1] provides what it describes as a Clinical Skills System that is a comprehensive digital solution for managing and operating a clinical skills center. The system has four major components: user management and content creation, exam management, scoring and reporting, and professional quality audio/video. This system attempts to address the same concerns about efficiency and automation in a surgical training environment. It uses a card swiping mechanism to identify residents and monitor their progress. It is mostly built on existing content management technology, and is not concerned with inferring context from sensed data.

More generally, to the best of our knowledge, none of the existing surgical training systems seek to infer significant events from the sensed data, or to use such data to infer the context of the surgical procedure and create a smart, adaptive surgical training space.

\section{OVERALL ARCHITECTURE}

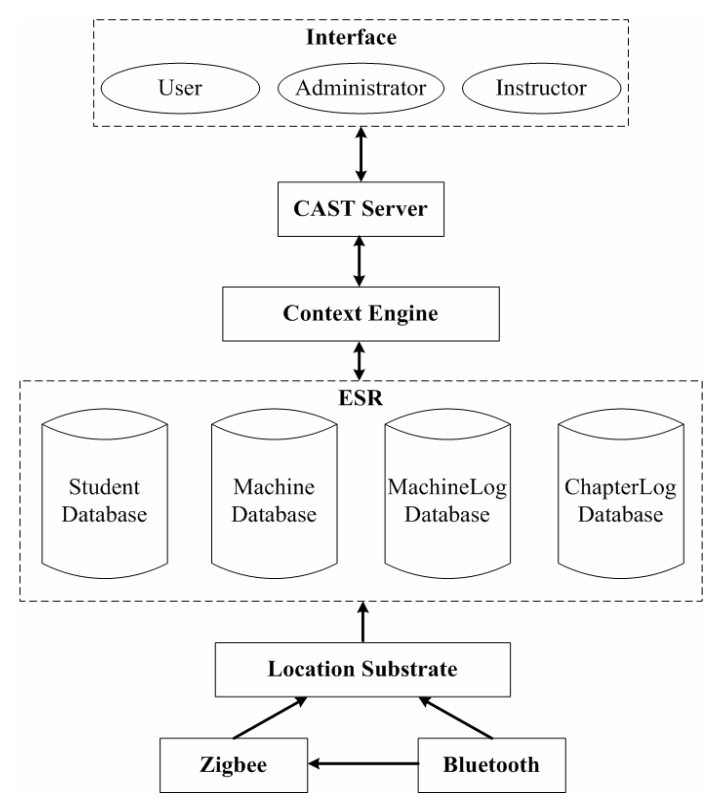

Figure 1. A high-level overview of the CAST system.

As in any ubiquitous computing system, location plays an important role in CAST. As shown in Figure 1, we use a location substrate consisting of a combination of Zigbee and Bluetooth to provide location information. This location information is then fed into a location database which keeps track of information such as which students were in front of a simulator and for how much time. The simulators in general 
require that students complete relevant chapters from the Fundamentals of Laparoscopic Surgery (FLS) training program before working on them. We host the FLS chapters on a web server that students can access through their logins. The chapters checked out are stored as part of the student's Electronic Student Record (ESR), which is updated when students check out chapters through the web interface. When the location substrate detects that a student is standing next to a simulator, it queries the student's context to verify that the student has completed the required FLS chapters. Only if the student has finished the required chapters is he/she allowed to work on the simulator.

Currently, information from sensors, such as training boxes, video recorders, RF tags, and cell phones, provide basic context information. These low level data streams are processed to generate higher-level primitive events, such as a resident entering the training room. A hierarchical knowledge-based event detection system correlates primitive events, resident data, and workflow data to infer high-level events, such as the finishing of a training module. Video streams of the training procedure are time-stamped and labeled with the inferred higher-level events. . These video recordings, location data, and performance data from simulators can be viewed offline by instructors. Moreover some simulator manufacturers are working on providing automated performance evaluation through video metric analysis. This resulting analysis, where available, could also be used as part of the ESR. The resulting ESR will provide trainers of physicians with a permanent record of the training session including an evaluation of the trainee's performance, the duration of the session, the number of times the trainee attempted the module before attempting the exam, and a labeled and time-stamped video of the session.

In a hospital setting with 10-20 residents, the smart training space will monitor the training activities of each resident more closely, and improve the workflow in the training center by allowing residents to sign up for a simulator at an allotted time only if they had the appropriate prerequisite tests/lessons and had been cleared by their mentor. Thus, a trainee surgeon may practice at a simulator during hours of convenience and be evaluated at the end of a session without the need of a trainer.

More generally, an important contribution of our system is that it makes the entire process of surgical training asynchronous. The instructor no longer needs to be physically present with the student during training. Many of the "adaptations" that a physically present instructor would have made (guiding the students to the right simulators and pointing out particular skills they needed to master, for instance) are now done by the system automatically. Moreover, the capture of the data stream from the simulators and the video of the trainee's hands as he/she practice on the laparoscopic simulators allow the evaluation to be done separately as well. This can also help remove the location dependence of surgical training. For instance, a student could do his training at any available simulation center (as long as it is networked with the parent school) and still have his/her procedure reviewed by the instructor back in the parent medical school, or even by an instructor at a different school.

CAST will also alleviate the burden of viewing the entire training video for evaluation even where automatic video metric analysis is not available or possible. It will provide the trainer with a labeled and time-stamped video of each training session that is correlated with the events signaled by the underlying simulator. For instance, the simulator may signal that the cut was made outside the designated area. Since the video timestamps will be correlated to the timestamps of the simulator output, the trainer can jump to portions of the video which are critical.

\section{LOCALIZATION}

Based on experiments conducted at the MASTRI, the Awarepoint $^{\mathrm{TM}}$ system, like most other commercially available location systems such as Ekahau [22], provides room level accuracy which suits the typical requirements of a hospital for asset or personnel tracking. However the CAST system requires finer localization to be able to place a student as being in a position to operate a particular simulator when there may be more than one in a room. We decided to use Bluetooth to provide localization at this granularity. As a result, our system uses a combination of Awarepoint and Bluetooth for localization.

\section{A. Awarepoint ${ }^{T M}$}

Awarepoint seeks to address the limitations in RFID technology and claims to have developed a real-time solution for one of hospitals' major problems, tracking of the movement of their staff, patients, and equipment. Awarepoint's tracking system is based on Zigbee, a high level communication protocol using the IEEE 802.15.4 standard for wireless network [16]. It is designed for radio frequency applications which require a low data rate, long battery life and secure networking. Awarepoint base stations, plugged directly into wall sockets, form a mesh network to deliver data from tags (such as signal strength and identifier) to a server which then uses a proprietary approach to identify the location of the tag. Each trainee is assumed to carry a tag. Awarepoint's standard user interface is a GUI that shows the location of the tags in a facility map. However, this is not appropriate for our purpose. As a part of a collaborative effort with Awarepoint, we have been provided access to their server database and associated SOAP interfaces so that we can directly query the location of a tag.

\section{B. Bluetooth Module}

We use Bluetooth to provide machine-level location information so that instructors can query for information such as how much time students spend in front of a machine. We periodically broadcast a Bluetooth device inquiry message, which returns the devices in range which respond to the inquiry. However, this method has high latency and does not necessarily return all Bluetooth devices in range, as some of them may not be listening on the same channel as the inquiry was sent and hence may not respond. As a result we decided to use a different approach. Our approach is motivated by the fact that we are not looking for "any" device but only for devices belonging to trainees. Each trainee is assumed to always have a Bluetooth capable device on him/her and the device addressstudent association is maintained in the student database. In our method, we periodically initiate connections to a list of MAC addresses obtained from the student database, and if the connection succeeds, we can infer that the corresponding device, and hence student, is in range. This method works well 
when the number of students is small, but the time to discover a device in this case grows with the number of students. To reduce the number of MAC addresses to initiate connections to, we use the Zigbee location information which provides room level accuracy, and initiate connections only to devices belonging to trainees currently in the room. When a single trainee is near a simulator, this suffices. However, since multiple trainees could be in the range of a machine, we still need a way to distinguish which one is actually using the machine. We achieve this by displaying a drop down list of students in range and requiring students to log in before using the machine. Thus we provide an additional layer of authentication, when Bluetooth discovery alone is unable to identify a student.

\section{FUNDAMENTALS OF LAPAROSCOPIC SURGERY (FLS)}

FLS is "a comprehensive, CD-ROM-based education module that includes a hands-on skills training component and assessment tool designed to teach the physiology, fundamental knowledge, and technical skills required in basic laparoscopic surgery. [14]." It was created by the Society of Gastrointestinal and Endoscopic Surgeons (SAGES) which is accredited by the Accreditation Council for Continuing Medical Education (A.C.C.M.E.) to sponsor Continuing Medical Education for physicians. To the best of our knowledge, FLS is the only CDbased education module that can be used to acquire CME credits. Since our system needs to incorporate the FLS curriculum and move away from its present CD based model, we need to obtain appropriate permissions. While this is being discussed, we have mocked up curriculum to represent the 14 modules in the FLS as shown in Figure 3.

\section{A. Webservice and MySql database}

We are hosting the mocked FLS curriculum on an Apache web server. We are using video clips to represent each of the modules. The user has to authenticate herself to the system to check out a training module. We track when students log in, for how long, which chapters they check out, in what sequence, and then log the information in the corresponding tables in the ESR. This information is useful in analyzing student progress. It also allows us to direct students to the tests they need to take and the procedures they need to practice on particular machines. So for instance if a trainee tries to use a simulator for which she has not checked out the appropriate lessons, we prevent her from using it.

\section{IMPLEMENTATION DETAILS}

The target development platform for CAST is the Nokia N800 [18]. The N800, pictured in Figure 2, has an impressive set of features such as Bluetooth, WiFi, and an inbuilt camera in a small form factor which makes it an attractive choice for capturing context in the training room. The N800 runs a Debian Linux distribution, which makes developing and porting applications easy. Each simulator has an associated N800 device. It serves as our Bluetooth location base stations. We use the built in camera to capture live video streams of students' training and relay it over $\mathrm{WiFi}$ to a central video database for indexing, potential automatic analysis, and review by the instructor. The N800 can also accept the simulator data feeds and stream them to the ESR. Our application code is mostly written in Python, using python for maemo [17]. N800's built in video chat, RSS feedreader, and podcast applications are also useful in allowing trainee-mentor interactions.

We have implemented Bluetooth localization using the PyBluez [19] module and BlueZ [20] stack on Linux. The Awarepoint server exports location information both through a database and a web service. We currently use the Awarepoint web service to obtain room level location information.

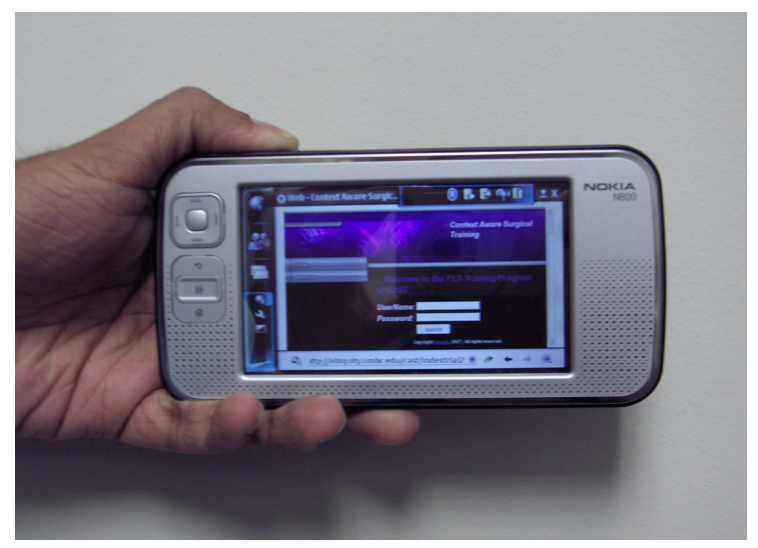

Figure 2. Login page to FLS Interface on Nokia N800.

The ESR is defined using RDF-S. Figure 4 gives a snapshot of the ESR in RDF-S. In the present implementation, we do not use a triple store. Instead, we have a preliminary version of the ESR as a MySQL database to allow for rapid prototyping. As we begin to reason over the sensed data more fully, we will migrate towards a triple store such as Jena [23].

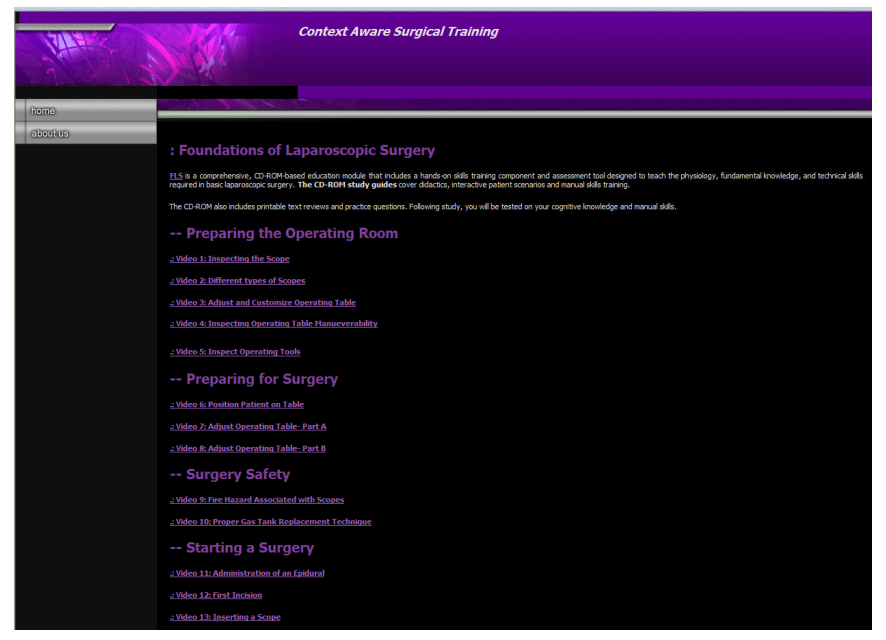

Figure 3. Mock FLS Curriculum

We have implemented and integrated the various modules of the system to create a prototype application. We can populate the student locations from the Awarepoint system, use Bluetooth to recognize trainees, and keep track of (mock) FLS modules checked out. We can also capture the video stream, although we do not yet synchronize it with the data stream captured from the simulators. This is because the simulator data 
streams are in proprietary formats for which no public documentation is available. We are presently working with some of the device manufacturers to understand their formats. One of the simulators used in the initial CAST deployment is shown in Figure 5.

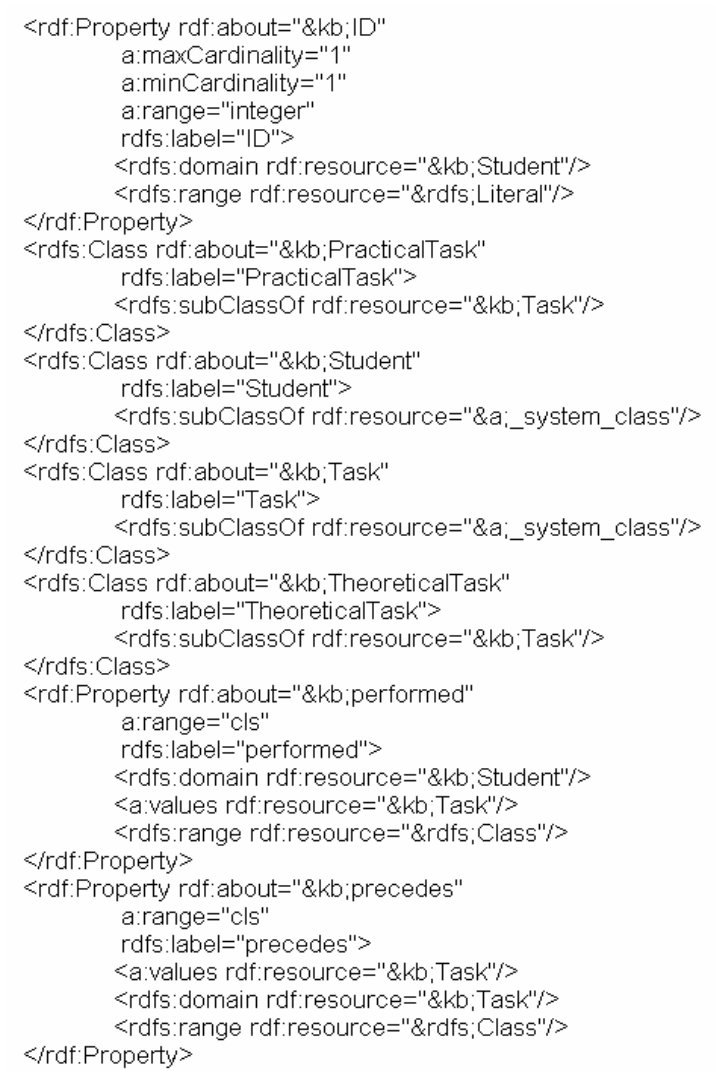

Figure 4. Snapshot of ESR in RDF-S.

\section{FUTURE WORK}

In the near future, we will evaluate the impact of such a context aware, ubiquitous system for surgical training in collaboration with colleagues at the University of Maryland Medical System. We will consider the security, effectiveness and efficiency of the system. Other simulators at MASTRI include a ProMIS ${ }^{\mathrm{TM}}$ surgical simulator as well as a METI $^{\mathrm{TM}}$ Human Patient Simulator ${ }^{\mathrm{TM}}$. The ProMIS ${ }^{\mathrm{TM}}$ surgical simulator includes performance metrics that have been validated for use with the SAGES FLS program that we intend to use in the ESR. The METI ${ }^{\mathrm{TM}}$ Human Patient Simulator ${ }^{\mathrm{TM}}$ provides a log of vital signs during a surgical training procedure. We aim to expand CAST to capture data from these sources as well.

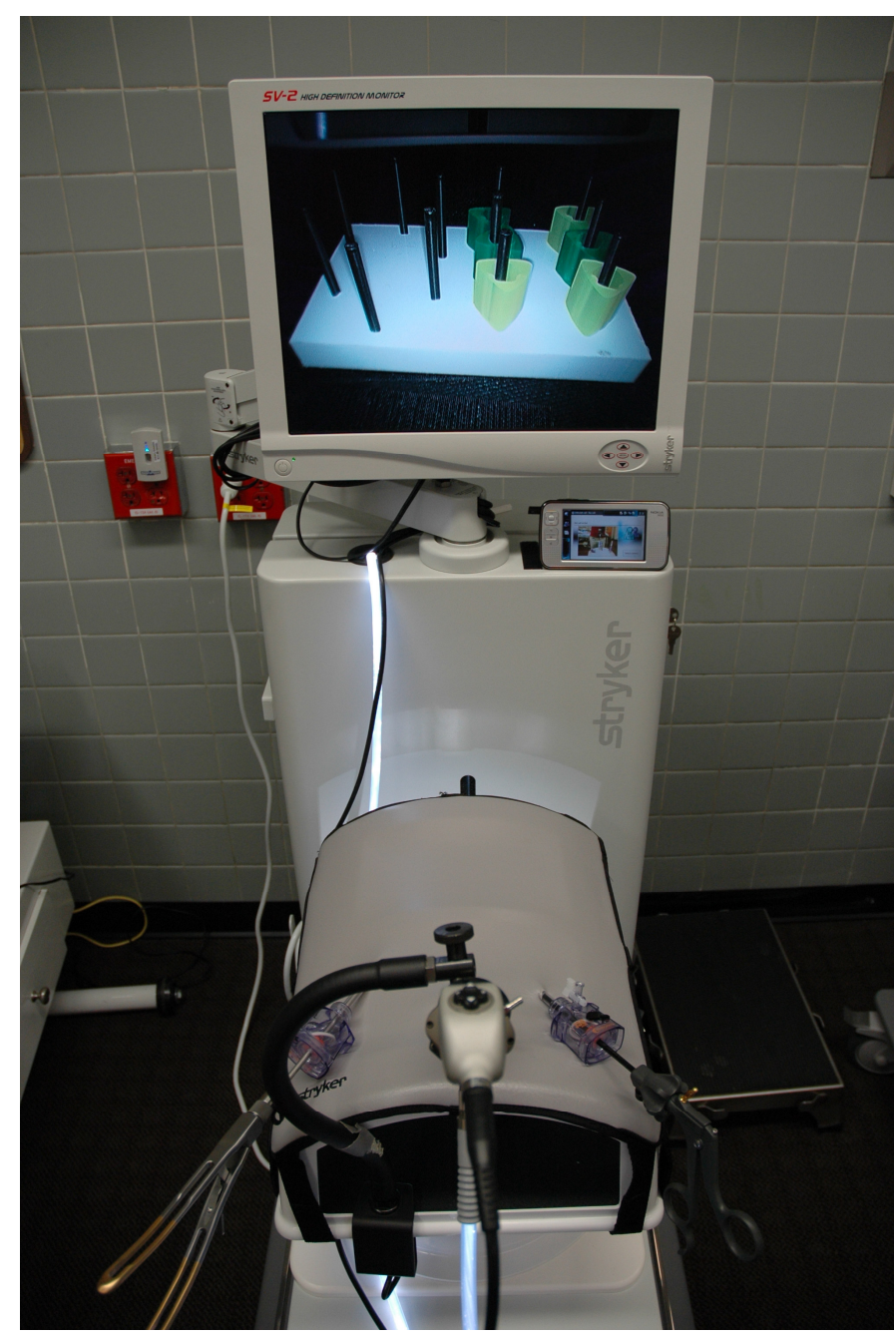

Figure 5. A Laparoscopic Training Simulator with an N800.

\section{REFERENCES}

[1] "Clinical Skills System", B-Line Medical: Comprehensive Digital Solutions, http://www.blinemedical.com/solution/clinicalskills.

[2] M.A Reznek, P. Harter, and T. Krummel, "Virtual reality and simulation: training the future emergency physician," Acad. Emer. Med., vol. 9, issue 1, pp. 78-87.

[3] M. . Fried, R. Satava, S. Weghorst, A.G. Gallagher, C. Sasaki, D. Ross, M. Sinanan, J.I. Uribe, M. Zeltsan, H. Arora and H. Cuellar, "Identifying and reducing errors with surgical simulation," Qual. Saf. Health Care, vol. 13, pp. 19-26, 2004.

[4] P.J. Gorman, A.H. Meier, T.M. Krummel, "Simulation and virtual reality in surgical education: real or unreal?," Arch. Surg., vol. 134, pp. 1203-1208, Nov 1999.

[5] M.J. Niederee, J.L. Knudtson, M.C. Byrnes, S.D. Helmer, R. S. Smith, "A survey of residents and faculty regarding work hour limitations in surgical training programs,"Qual. Saf. Health Care, vol. 13, pp. 19-26.

[6] F. H Garlick, "Surgical training of doctors in their own isolated hospital,” Aust. N.Z. J. Surg., vol. 70, pp. 456-458, 2000.

[7] S. Grange, "A virtual university infrastructure for orthopaedic surgical training with integrated simulation," PhD, Engineering, Mathematics and Computing, University of Exeter, United Kingdom, 2006.

[8] K. Kalicki, F. Starzynski, A. Jenerowicz, K. Marasek, "Simple ossiculoplasty surgery simulation using haptic device," тие, pp. 932936, 2007 International Conference on Multimedia and Ubiquitous Engineering (MUE'07), 2007. 
[9] G. Welch, R. Yang, B. Cairns, H. Towles, A. State, A. Ilie, S. Becker, D. Russo, J. Funaro, D. Sonnenwald, K. Mayer-Patel, B. D. Allen, H. Yang, E. Freid, A. van Dam, and H. Fuchs, "3D Telepresence for Off-Line Surgical Training and On-Line Remote Consultation," Proceedings of ICAT CREST Symposium on Telecommunication, Teleimmersion, and Telexistence, The University of Tokyo, Tokyo, Japan, December 2004. Invited submission.

[10] G. Welch, R. Yang, S. Becker, A. Ilie, D. Russo, J. Funaro, A. State, K.Low, A. Lastra, H. Towles, B. Cairns, H. Fuchs, and A. van Dam. "Immersive Electronic Books for Surgical Training, “ IEEE Multimedia, vol. 12, no. 3, pp. 22-35, July-September 2005.

[11] A. Ilie, k. Low, G. Welch, A. Lastra, H. Fuchs and B. Cairns, "Combining Head-Mounted and Projector-Based Displays for Surgical Training," Presence: Teleoperators and Virtual Environments, vol. 13, no.2, pp. 128-145, April 2004.

[12] J.C. Rosser, Jr., P.J. Lynch, L. Cuddihy, D.A. Gentile, J. Klonsky, R. Merrell, "The impact of video games on training surgeons in the $21^{\text {st }}$ century," Arch. Surg., vol. 142, no. 2, pp. 181-186, Feb 2007.

[13] "The Awarepoint ${ }^{\mathrm{TM}}$ Solution", Awarepiont: Real-time Awareness Solutions, http://awarepoint.com/Healthcare/Solution.html.
[14] "What is FLS", Fundamentals of Laparoscopic Surgery, http://www.flsprogram.org/.

[15] "OR of the Future", University of Maryland Medical Center, http://www.umm.edu/center/or_of_future.htm.

[16] "Zigbee", Wikipedia, http://en.wikipedia.org/wiki/Zigbee.

[17] "Maemo", http://maemo.org/

[18] "Nokia N800", http://www.nseries.com/n800/.

[19] "PyBluez", http://org.csail.mit.edu/pybluez

[20] "BlueZ', http://www.bluez.org/

[21] "RDF-S", http://www.w3.org/TR/rdf-schema/

[22] "ekahau", http://ekahau.com/

[23] "Jena-A Semantic Web Framework for Java", http://jena.sourceforge.net

[24] "ProMIS"M surgical simulator", http://www.haptica.com/id11.htm

[25] "Human Patient Simulator", METI Medical Education Technologies, Inc., http://www.meti.com/Product_HPS.html 